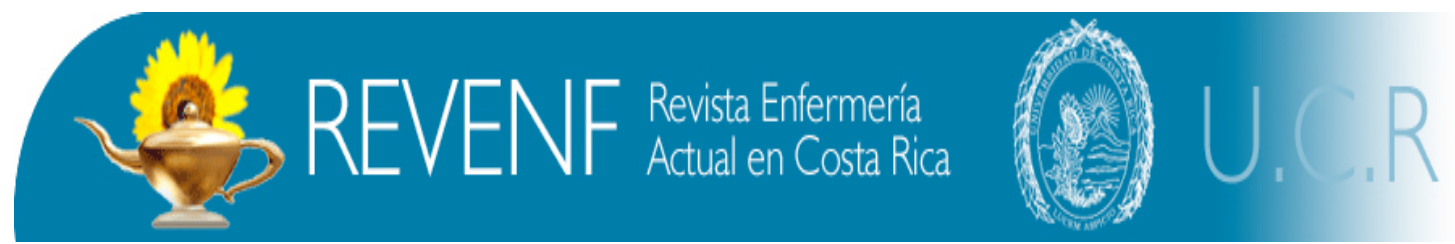

Revista Semestral Número 17 Octubre 2009- Marzo 2010

ISSN 1409-4568

\title{
FACTORES SOCIODEMOGRÁFICOS MATERNOS QUE PREDISPONEN A LA PRESENCIA DE SÍFILIS CONGÉNITA EN EL NEONATO ${ }^{1}$
}

\section{COMO CITAR ESTE ARTÍCULO}

Morales Sánchez, Adriana. Factores sociodemográficos maternos que predisponen a la presencia de sífilis congénita en el neonato. Rev. Enfermería Actual en Costa Rica [en línea].2009, No.17 [citado (fecha)]. Disponible World Wide Web: $<$ http://www.revenf.ucr.ac.cr/sifilis.pdf> ISSN 1409-4568

Adriana Morales Sánchez ${ }^{2}$

\begin{abstract}
RESUMEN
Este artículo presenta los principales resultados de uno de los objetivos específicos de la investigación denominada "Reconstrucción de la Atención de Enfermería al neonato con prueba sanguínea VDRL positiva y diagnóstico presuntivo de sífilis congénita que incluya a los progenitores, en el Servicio de Neonatología del Hospital de las Mujeres Dr. Adolfo Carit Eva”. Surgió la inquietud investigativa por la observación en la práctica clínica cotidiana, en donde se aprecia un aumento en el número de casos de neonatos atendidos e ingresados con el diagnóstico de "sífilis congénita" en las salas de neonatología; por lo cual deben permanecer por largos periodos de tiempo - de 15 días a un mes- recibiendo tratamiento. Además, por el cambio en el perfil epidemiológico que presenta actualmente Costa Rica, con respecto al aumento en los índices de enfermedades de transmisión sexual, según lo señala Alburg (2008). Se utilizó un diseño cuantitativo, de tipo descriptivo y transversal. La población estuvo conformada por 2346 mujeres atendidas por parto de las cuales se seleccionó una muestra de 69 mujeres estudiadas por sífilis, así como una muestra de 61 neonatos tamizados por sífilis congénita.

El resultado relevante en este estudio radicó en el desconocimiento por parte de las mujeres gestantes sobre la sexualidad, el bajo nivel educativo y baja autoestima, así como otros factores de índole cultural, social y personal que las hace más vulnerables ante esta problemática de salud pública.
\end{abstract}

Palabras claves: sífilis, sífilis congénita, infección madre-hijo, factores de riesgo, perfil de riesgo.

\footnotetext{
${ }^{1}$ Fecha de recepción: febrero 2009

Fecha de aceptación: junio 2009

${ }^{2}$ Licenciada en Enfermería. Estudiante de la maestría en Enfermería Ginecológica, Obstétrica y Perinatal de la Universidad de Costa Rica. Correo electrónico: nanitamor2026@hotmail.com
} 


\begin{abstract}
This article presents the main results of one of the specific objectives of the research study "Reconstruction the nursing care to the newborn with positive VDRL blood test and presumptive diagnosis of congenital syphilis involving parents in the Neonatology Service of Hospital Dr. Adolfo Carit Eva.

Investigative concerns emerged by observation in everyday clinical practice, where it has been an increase in the number of cases of infants treated and admitted with a diagnosis of "congenital syphilis" in neonatal nurseries, so they must stay for long time-periods of 15 days to a month receiving treatment. Furthermore, the change in the epidemiological profile now featuring Costa Rica, with respect to increased rates of sexually transmitted diseases, as indicated Alburg (2008).

By using a quantitative, descriptive and transversal. The population consisted of 2346 women attended by delivery of which selected a sample of 69 women screened for syphilis, and a sample of 61 infants screened for congenital syphilis.

The relevant result in this study lay in the ignorance of pregnant women about sex, low education and low self-esteem, as well as other factors, including cultural, social and personal which makes them more vulnerable to this public health problema.
\end{abstract}

Keywords: syphilis, congenital syphilis, mother-child infection, risk factors, risk profile.

\title{
INTRODUCCIÓN.
}

Este artículo consiste en una recopilación de información para destacar cuales son los principales factores de riesgo perinatales, que se relacionan con el diagnóstico de sífilis congénita, dicha investigación incluyó estadísticas nacionales y del centro hospitalario participante en el estudio, el Hospital de las Mujeres Dr. Adolfo Carit Eva, asimismo con la utilización de fuentes primarias y expedientes de salud de las usuarias y neonatos atendidos durante los meses de mayo a diciembre del 2008.

En los últimos años se ha descuidado la vigilancia de esta enfermedad, desde el momento en el que se descubre su tratamiento, dando más auge a otra enfermedad de transmisión sexual, como lo es el virus de inmunodeficiencia humana adquirida, que si bien es cierto es mortal, no es la única que ocasiona muerte o trastornos a largo plazo en un ser humano. (Indicadores de Salud y Bienestar, OPS, 2004).
Además, se creía que la sífilis estaba erradicada, (Calvo, 2005), algo contrario a lo encontrado en este estudio. Es por esta razón que se consideró relevante hacer un análisis epidemiológico del evento en mención, e identificar los factores relacionados a su ocurrencia.

El estudio surgió debido a que en el Servicio de Neonatología de este Centro de Salud, es frecuente observar ingresos de neonatos infectados con sífilis materna, que ocasionan prolongados periodos de internamiento para recibir tratamiento y evitar complicaciones más severas. Además actualmente este Hospital, no cuenta con protocolos, normas o investigaciones previas, en las cuales se estudien los anteriores factores de riesgo, por lo que se pretende, contribuir en ofrecer información a este centro de salud, y que enfermería a través de estos datos, brinde una alternativa de atención, donde se apliquen los lineamientos de educación para la 
prevención, detección, diagnóstico, tratamiento, seguimiento y rehabilitación durante esta enfermedad, a partir de los perfiles que se investigan y principales poblaciones de riesgo.

La sífilis congénita es una enfermedad, que se trasmite al niño y la niña, por medio de la madre infectada con sífilis, que conlleva múltiples riesgos para el nuevo ser, los cuales pueden ser mortales. (Kumar, 2003).

La transmisión de la sífilis ocurre en el útero pero las manifestaciones clínicas aparentes en el fruto de la gestación son muy variables determinando el período de incubación clínico. El paso del Treponema Pallidum, al feto puede ocurrir en cualquier momento del embarazo, pero el daño se produce después del 4 mes, coincidiendo con el inicio de la respuesta inmune fetal. (Orozco, 2003).

La severidad se relaciona con el momento en que la madre adquirió la infección y, por lo tanto con los estadios de infección materna que cursan durante el embarazo, la carga de Treponemas que infectan al feto y la oportunidad de la respuesta inmunológica de la madre. Antes de la concepción o muy precozmente durante el embarazo, la respuesta inmunológica humoral de la madre, disminuye la carga total de Treponemas que alcanza al feto y, por lo tanto el daño fetal. Se presentan infecciones más leves o no se produce infección fetal. (Schwarzc, 2005).

A nivel internacional, la Organización Panamericana de la Salud la OPS en una investigación denominada: Eliminación de Sífilis Congénita en América Latina y el Caribe, (Julia Valderama, 2003):
Se expone que en América Latina y el Caribe existen $\mathbf{3 3 0 . 0 0 0}$ mujeres embarazadas que tienen una prueba positiva para sífilis, y no reciben tratamiento durante el control prenatal. Aunque el estadio de la enfermedad es un factor determinante, se estima que de estos embarazos nacen 110.000 niños con sífilis congénita, y un número similar resulta en aborto espontáneo. (p. 11)

Partiendo del hecho de que es la madre quien trasmite la enfermedad al neonato, (Lukehart, 2005), este objetivo específico de estudio pretendió determinar, los factores socio demográficos que se asocian a la adquisición de la enfermedad por parte materna, para determinar un perfil de riesgo en la población.

De manera directa, el estudio beneficia a las futuras gestantes, ya que conociendo cuales son los factores de riesgo que hacen a estas mujeres no recibir atención oportuna, o contraer la enfermedad se tendrá una base para atacar este foco, y se conocerá el perfil de estas gestantes, para tratarlas y evitar entonces que el neonato salga infectado.

Aplicando los nuevos conceptos de proceso de salud-enfermedad, este estudio tomó en cuenta cuatros aspectos enfocados en la dimensión biológica, conciencia y conducta, ecológica-entorno, y dimensión económica, estas fueron subdivididas según factores de riesgo expuestas por la Dra. Susan Vandale Toney y Dr. Felipe Uribe, del Centro de Investigaciones en Salud Pública, del Instituto Nacional de Salud Pública, de Cuernavaca, Morelos, donde consideran que los factores prevalentes en la adquisición de sífilis se orientan en los factores sociales como los son: la limitada capacidad de negociación de la relación sexual; el poco control y la baja 
capacidad de juicio para protegerse de la transmisión sexual; durante la relación sexual; la baja autoestima, para ser aceptada en un grupo determinado; así como su estado civil. Con respecto a los factores culturales se encuentran: el conflicto de género; el machismo predominante en esta cultura; la falta de educación sexual adecuada y oportuna por perjuicios culturales y religiosos; el inicio de relaciones sexuales a temprana edad. Entre los factores personales están: el contacto sexual de riesgo; las conductas sexuales riesgosas; la presencia de múltiples compañeros sexuales en el pasado o en la actualidad; enfermedad de transmisión sexual de cualquier tipo en la actualidad o en el pasado; el consumo de drogas psicoactivas (marihuana, semillas de marihuana, cocaína, heroína, alcohol, entre otros; así como la ausencia de atención prenatal oportuna y adecuada es el factor más importante en la incidencia de sífilis congénita. Y como factores económicos se encuentran: el nivel socioeconómico bajo; el bajo nivel educativo, pues existe un menor nivel de educación sexual, y un desconocimiento del riesgo en que se incurre; la baja cobertura en los servicios de salud; el desconocimiento de que atención prenatal es gratuita por decreto constitucional del país; la dependencia económica de un proveedor que promueva la incidencia al abuso; la actividad comercial sexual.

El objetivo general del esta recopilación de datos, fue determinar los factores socios demográficos maternos, asociados al diagnóstico de sífilis congénita, en neonatos atendidos en el Servicio de Neonatología del Hospital de las Mujeres de mayo a diciembre del 2008.

\section{CONSIDERACIONES ETICAS.}

Esta investigación se rigió bajo los principios generales estipulados en el Artículo 4 del Capítulo I de disposiciones generales del reglamento para la investigación biomédica en los servicios asistenciales de la Caja Costarricense de Seguro Social, en el cual, se reconocen como principios generales que deberán regir la materia: el respeto a la dignidad de las personas, la beneficencia, la no maleficencia, la autonomía y la justicia distributiva.

Los progenitores, estuvieron en todo su derecho de aceptar o no participar en el estudio, regidos bajo su propia autodeterminación, y se respetó al máximo si tenían una autonomía disminuida, ayudándolos en todo momento y nunca forzando a la participación en el estudio, previó a su participación, se obtuvo el debido consentimiento informado.

Por medio de esta investigación se pretendió en todo momento obtener beneficios para los participantes, y contribuir a ofrecer una mejor atención en estos casos. El riesgo de la participación fue mínimo ya que la única intervención con el participante fue a la hora de llenar el cuestionario, de forma que el único beneficiado fueron el progenitor $y$ neonato. La práctica enfermera en este caso se orientó a hacer las cosas lo mejor posible.

Durante el proceso de la investigación toda la población de estudio fue trata de manera igualitaria y equitativa, sin distinción de etnia, género o limitante externa como el ser analfabeta. Así mismo no se faltó el respeto de ningún participante y se trabajo bajo un ambiente de respeto y armonía. 
En ningún momento la investigación pretendió hacer daño físico, emocional o psicológico a ningún participante, sin embargo en dos casos las madres al realizar el cuestionario, entraron en un insight, y presentaron un estado de ansiedad relacionado con el diagnóstico de su hijo, estas mujeres se refirieron a prontitud con miembros especializados del equipo interdisciplinario del hospital de estudio, para solventar cualquier dificultad. Y por ser este diagnóstico un problema social, la mayor parte de las mujeres fueron referidas tanto a trabajo social como a psicología como rutina.

Para evitar la ruptura de la confidencialidad de la información recolectada, se utilizaron medidas extremas para evitar esta situación, dentro de las acciones que garantizaron la confidencialidad están: la no necesidad de datos personales como nombre, cedula, teléfonos. Además, los datos fueron recolectados únicamente por la investigadora, la cual custodiara los cuestionarios de categorías de análisis, así mismo los datos obtenidos serán presentados como un conjunto de datos estadísticos generales, garantizando de esta forma la total confidencialidad.

\section{MATERIALES Y METODOS.}

Este artículo se fundamentó en el patrón de conocimiento empírico, (Hernández Sampierie, 1998), ya que describe y predice a través de la observación y repetición de los fenómenos referentes a la adquisición de la sífilis materna y sífilis congénita, además debido a que utiliza los componentes básicos de Enfermería como disciplina, por medio de la práctica diaria realizada en el servicio de neonatología, donde a través de esta, se evidencia el aumento en el número de casos por lo cual surge la inquietud investigativa.
Se realizó un estudio cuantitativo, descriptivo-transversal, realizado en el Hospital de las Mujeres Dr. Adolfo Carit Eva.

La definición de caso, (Barrantes, 1999), fue: Toda mujer atendida para su parto y neonato nacido, atendidos en el Hospital de las Mujeres Dr. Adolfo Carit Eva, de mayo a diciembre del 2008.

La población de estudio fue: todo binomio madre - hijo, atendidos por parto y nacimiento, siendo un total de 2346 Para este estudio las dimensiones correspondieron a: factores sociales, económicos, culturales y personales que inciden en las implicaciones de riesgo para padecer de sífilis materna, provocando riesgos perinatales, los cuales se compararon con población que enfrentó la misma situación y no presentó la enfermedad. Se analizaron variables sociodemográficas propias de la Caracterización de la población como son: edad, residencia, estado civil, profesión u oficio y escolaridad.

Para la recolección de datos se utilizó el procesamiento manual, una vez obtenidos los datos requeridos por la unidad de estadística, se procedió a revisar cada una de las mujeres, se analizó una muestra de 69 estudiadas por sífilis. Así como 2346 neonatos, de los cuales se analizó una muestra de 61 tamizados por sífilis congénita.

La fuente de datos fue proporcionada en su mayoría por la unidad de estadísticas del hospital participante en el estudio, y de bases de datos de la investigadora al trabajar con estas mujeres mediante la formulación de preguntas generadoras, así como la aplicación de instrumentos realizados Centro Latinoamericano de 
Perinatología (CLAP), expediente personal de la usuaria y neonato.

los expedientes de casos reportados como positivos por sífilis materna y congénita, extrayendo las variables de interés para el estudio.

El procesamiento de información, se realizó una vez obtenidos los datos en una base común, fueron analizados de manera individual, mediante la estadística básica descriptiva y las medidas de tendencia central. Luego fueron agrupados en frecuencias y analizados relacionando las variables de interés, por medio de casos y controles para poder definir un riesgo probable mediante la utilización de odds estadísticos. (Dever, 1991).

\section{RESULTADOS.}

En la tabla 1, sobre edad materna, se observó que la edad media de las mujeres participantes en el estudio fue de 19 a 23 años, con un rango total entre 14 y 45 años. Además que la población adolescente representa un número importante de embarazo y de presencia de esta enfermedad. Se observó que conforme avanza la edad materna, disminuye el riesgo de padecer la enfermedad, ya que en mujeres mayores de 29 años únicamente se presentaron 13 casos de los 69 tamizados.

Tabla 1

San José: Distribución de casos por sífilis en embarazadas según edad,

\begin{tabular}{|c|c|c|c|c|}
\hline $\begin{array}{c}\text { Edad } \\
\text { en años }\end{array}$ & $\begin{array}{c}\text { Con } \\
\text { sífilis }\end{array}$ & $\%$ & Sin sífilis & $\%$ \\
\hline 14 a 18 & 24 & 35 & 526 & 23.10 \\
\hline 19 a 23 & 17 & 24.6 & 640 & 28.10 \\
\hline 24 a 28 & 15 & 21.7 & 618 & 27.14 \\
\hline 29 a 33 & 5 & 7.2 & 398 & 17.5 \\
\hline 34 a 39 & 5 & 7.2 & 85 & 3.73 \\
\hline Más de 40 & 3 & 4.3 & 10 & 0.44 \\
\hline Total & 69 & 100 & 2277 & 100 \\
\hline
\end{tabular}

En la tabla 2, sobre distribución geográfica, se observó que aplicadas las

medidas de tendencia central, se dedujo que el promedio de casos de sífilis materna por distrito es de 4 casos, la media se encuentra en 3 casos por distrito y la moda se encuentra repetida en 1 y 6 casos por distrito. Además que con un $41 \%$ de casos el cantón de Desamparados, presentó mayor ocurrencia, y de este cantón, el distrito con mayor número de casos, fue Patarrá, con el doble de casos comparado con distritos como Desamparados centro y San Miguel. El segundo cantón con mayor incidencia se representa en el Central, siendo el distrito de Hatillo y San Sebastián, los que presentan mayor número de casos. Además que la zona que presenta menor incidencia de las áreas de atracción corresponde a Curridabat.

Tabla 2

San José: Distribución de mujeres con diagnóstico de sífilis que tuvieron parto en el Hospital de las Mujeres, según lugar de residencia, 2008.

\begin{tabular}{|c|c|c|c|}
\hline CANTON & DISTRITO & CASO & TOTAL \\
\hline \multirow[t]{7}{*}{ Central } & Zapote & 1 & \\
\hline & San & 6 & \\
\hline & Sebastián & & \\
\hline & San & 2 & 20 \\
\hline & Francisco & 3 & \\
\hline & Uruca* & 8 & \\
\hline & Hatillo* & & \\
\hline \multirow{7}{*}{$\begin{array}{c}\text { Desampara } \\
\text { dos } \\
\end{array}$} & Desamparad & 6 & \\
\hline & os & & \\
\hline & San Miguel & 5 & \\
\hline & San Juan de & 1 & 27 \\
\hline & Dios & & \\
\hline & San Rafael & 3 & \\
\hline & Patarra & 12 & \\
\hline \multirow[t]{3}{*}{ Acosta } & San Ignacio & 1 & \\
\hline & Palmichal & 2 & 5 \\
\hline & Guatil & 2 & \\
\hline Aserrí & Aserrí & 6 & 6 \\
\hline Curridabat & Sabanilla & 1 & 1 \\
\hline Alajuelita & Alajuelita & 6 & 10 \\
\hline * & Concepción & 4 & \\
\hline Total & & & 69 \\
\hline
\end{tabular}

*Estas zonas no pertenecen al área de atracción del hospital participante en el estudio. 
Según la tabla 3, sobre las variables que se refieren a factores sociales, las mujeres con pareja estable (casadas y en unión libre) representado por un $56 \%$ de la población, tienen un mayor riesgo de padecer sífilis y contagiar a su bebé, que aquellas sin pareja estable (solteras, viudas, divorciadas), para un 42\%. Así mismo la relación sobre factores sociales, la valoración de autoestima, juega un papel importante es este estudio ya que un 99\% de la población, tenía un valoración de psicología que indicaba tener baja o moderada autoestima solo un $1 \%$ tenía autoestima alta.

Tabla 3

San José: Descripción de mujeres atendidas por parto en el Hospital de estudio, según estado civil y valoración psicológica, 2008.

\begin{tabular}{|c|c|c|c|c|}
\hline $\begin{array}{r}\text { Aspectos } \\
\text { sociales }\end{array}$ & $\begin{array}{r}\text { Con } \\
\text { sífilis }\end{array}$ & $\%$ & $\begin{array}{r}\text { Sin } \\
\text { sífilis }\end{array}$ & $\%$ \\
\hline $\begin{array}{rrr}\text { a. } & \text { Estado civil } \\
\text { - } & \text { Soltera } \\
& \text { Casada } \\
& \text { - } & \text { Viuda } \\
\text { - } & \text { Divorciada } \\
\text { - } & \text { Unión libre } \\
& - & \text { Otros }\end{array}$ & $\begin{array}{r}27 \\
13 \\
1 \\
3 \\
25 \\
0\end{array}$ & $\begin{array}{r}39.1 \\
18.8 \\
1.4 \\
4.3 \\
36.2 \\
0\end{array}$ & $\begin{array}{r}820 \\
351 \\
108 \\
28 \\
970 \\
0\end{array}$ & $\begin{array}{r}36 \\
15.5 \\
4.7 \\
1.2 \\
42.6 \\
0\end{array}$ \\
\hline 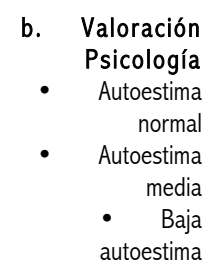 & $\begin{array}{l}20 \\
33\end{array}$ & $\begin{array}{l}23 \\
29 \\
48\end{array}$ & $\begin{array}{r}1288 \\
971 \\
18\end{array}$ & $\begin{array}{r}56.5 \\
42.6 \\
0.8\end{array}$ \\
\hline
\end{tabular}

En la tabla 4, sobre factores de riesgo culturales, el grado de estudios o nivel educativo, fue otra de las evidencias encontradas, donde, el tener bajo nivel educativo predispone a padecer de esta enfermedad.

Se evidenció que la no protección durante el acto sexual, constituye un riesgo en la adquisición de la sífilis con un $82 \%$ de probabilidad.
El mayor número de casos de sífilis materna se encontró en mujeres primigestas.

Con respecto a la edad de inicio de relaciones sexuales de las mujeres con sífilis se encontró que la edad promedio del inicio de sexualidad fue de antes o igual a los 15 años con un $42 \%$.

Tabla 4

San José: Descripción de mujeres atendidas por parto en el Hospital de estudio, según aspectos culturales

\begin{tabular}{|c|c|c|c|c|}
\hline $\begin{array}{l}\text { Aspectos } \\
\text { culturales }\end{array}$ & $\begin{array}{l}\text { Con } \\
\text { sífilis }\end{array}$ & $\%$ & $\begin{array}{l}\text { Sin } \\
\text { sífilis }\end{array}$ & $\%$ \\
\hline \multicolumn{5}{|l|}{ Nivel de estudios } \\
\hline Ninguno & 6 & 8.7 & 115 & 5.05 \\
\hline Primaria & 25 & 36.2 & 325 & 14.3 \\
\hline Secundaria & 30 & 43.5 & 626 & 27.5 \\
\hline Universidad incompleta & 8 & 11.6 & 905 & 39,7 \\
\hline Universidad completa & 0 & 0 & 306 & 13.4 \\
\hline \multicolumn{5}{|l|}{$\begin{array}{l}\text { Uso de métodos } \\
\text { anticonceptivos }\end{array}$} \\
\hline Si uso & 3 & 4.35 & 1085 & 82.8 \\
\hline No usa & 66 & 95.65 & 1192 & 17.2 \\
\hline \multicolumn{5}{|l|}{$\begin{array}{l}\text { Inicio de relaciones } \\
\text { sexuales }\end{array}$} \\
\hline Antes de 15 años & 33 & 47.8 & 963 & 42.3 \\
\hline De 15 a 20 años & 26 & 37.7 & 714 & 31.3 \\
\hline Adulto más de 20 & 10 & 14.5 & 600 & 26.3 \\
\hline
\end{tabular}

Observando los hallazgos de la tabla 5 sobre el nivel económico la población con ingresos menores a 120 mil colones representa índices de pobreza y predisposición a esta enfermedad ya que un $82 \%$ de la población se considera como pobre.

Dentro de estos factores de riesgo económicos mostrados, la ausencia de control prenatal, constituye un factor de riesgo importante representado por un 67 $\%$ de la población que no tuvo control y adquirió la enfermedad. Otro resultado relevante es que la mayoría de las mujeres son amas de casa dedicadas a su hogar. 
Tabla 5

San José: Descripción mujeres atendidas por parto hospitalario, Según aspectos económicos, 2008.

\begin{tabular}{|c|c|c|c|c|}
\hline $\begin{array}{l}\text { Aspectos } \\
\text { económicos }\end{array}$ & $\begin{array}{l}\text { Con } \\
\text { sífilis }\end{array}$ & $\%$ & $\begin{array}{l}\text { Sin } \\
\text { sífilis }\end{array}$ & $\%$ \\
\hline \multicolumn{5}{|l|}{$\begin{array}{l}\text { Ingresos mensuales en } \\
\text { colones }\end{array}$} \\
\hline Menos de 80.000 & 30 & 43.5 & 720 & 36.2 \\
\hline De 80.000 a 120.000 & 25 & 36.2 & 600 & 46.06 \\
\hline De 120.000 a 200.000 & 10 & 14.5 & 673 & 14 \\
\hline Más de 200.000 & 4 & 5.8 & 284 & 3.7 \\
\hline \multicolumn{5}{|l|}{ Ocupación de la mujer } \\
\hline Labores del hogar & 33 & 47.8 & 258 & 11.3 \\
\hline Propio & 5 & 7.5 & 215 & 9.44 \\
\hline Comercio & 10 & 14.5 & 785 & 34.47 \\
\hline Administrativo & 8 & 11.3 & 111 & 4.9 \\
\hline Estudia & 2 & 2.9 & 905 & 39.7 \\
\hline Comercio sexual & 11 & 16 & 3 & 0.13 \\
\hline \multicolumn{5}{|l|}{ Tipo de vivienda } \\
\hline Casa propia & 10 & 14.5 & 896 & 39.4 \\
\hline Casa alquilada & 25 & 36.3 & 1074 & 47.2 \\
\hline Prestada familiar & 20 & 28.9 & 270 & 11.85 \\
\hline Precario & 14 & 20.3 & 37 & 1.6 \\
\hline \multicolumn{5}{|l|}{ Control prenatal } \\
\hline $\mathrm{Si}$ & 23 & 33.4 & 1607 & 88.2 \\
\hline No & 43 & 66.6 & 670 & 11.8 \\
\hline
\end{tabular}

En relación a la siguiente tabla 6, sobre aspectos personales, se evidenció que casi la mitad de la población de mujeres con sífilis no planeó su embarazo en un $48 \%$, solo 21 mujeres consideraron que su embarazo si fue planeado, así mismo las mujeres con sífilis usaron drogas en algún momento de su vida para un $69 \%$.

Tabla 6

San José: Distribución de mujeres atendidas por parto hospitalario, Según aspectos personales, 2008.

\begin{tabular}{|c|c|c|c|c|}
\hline $\begin{array}{l}\text { Aspectos } \\
\text { personales }\end{array}$ & $\begin{array}{l}\text { Con } \\
\text { sífilis }\end{array}$ & $\%$ & $\begin{array}{l}\text { Sin } \\
\text { sífilis }\end{array}$ & $\%$ \\
\hline $\begin{array}{l}\text { Planeación del } \\
\text { embarazo }\end{array}$ & & & & \\
\hline $\mathrm{Si}$ & 21 & 30.4 & 1545 & 94.2 \\
\hline No & 48 & 69.6 & 732 & 5.8 \\
\hline Uso de drogas & & & & \\
\hline Si uso & 48 & 69.6 & 345 & 15.1 \\
\hline No usa & 21 & 30.4 & 1932 & 84.9 \\
\hline $\begin{array}{l}\text { Compañeros } \\
\text { sexuales }\end{array}$ & & & & \\
\hline 1 a 2 & 12 & 17.4 & 926 & 40.7 \\
\hline 3 a 4 & 42 & 60.8 & 1231 & 54 \\
\hline Más de 4 & 15 & 21.7 & 120 & 5.3 \\
\hline
\end{tabular}

De la población total de mujeres con sífilis, se encontró que 61 mujeres dieron a luz a un neonato con sífilis congénita, a 8 neonatos se les dio salida por VDRL no reactivo o débil, que a los 8 días durante la consulta de seguimiento a 5 se les reingreso por presentar VDRL reactivos, 3 de estos neonatos no fueron llevados a consulta posnatal y se desconoce su estado entorno a sífilis congénita.

Con base en los anteriores resultados, se elaboro un lista de factores de riesgo, con su condición de riesgo, en donde se evidenció mayores observaciones, comparados con aquellas mujeres que se enfrentaron a las mismas condiciones de parto y no presentaron la enfermedad, creando un perfil de riesgo para esta población de estudio, donde se encontró como muestra la tabla 7 que, la probabilidad de tener sífilis materna y vivir en el cantón desamparados es el doble con respecto a los que viven en el cantón central. La probabilidad de vivir en el distrito de Patarrá es de 4 veces mayor comparado con los otros distritos de desamparados, de padecer de sífilis materna durante el embarazo.

El riesgo de padecer de sífilis materna si se tiene 18 años o menos es de 1.9 veces más probable, casi el doble si tiene más de 18 años de edad.

El riesgo de padecer de sífilis materna siendo primigesta es 1,75 veces más probable que él no serlo.

El riesgo de tener pareja estable (casada o unión libre) es de 1.12 veces mayor que si no se tiene pareja (solteras, viudas, divorciadas).

El tener una baja autoestima presenta 4 veces más probabilidad de padecer sífilis 
materna que las que tienen una buena autoestima.

El tener un nivel educativo bajo (ninguno o primaria), presenta una probabilidad de 3 veces mayor que las mujeres con nivel educativo alto.

El no usar métodos anticonceptivos o de barrera, representó 20 veces más riesgo de padecer sífilis, que en las que usaron protección.

La probabilidad de padecer sífilis en inicios tempranos de relaciones sexuales es de 1.44 veces mayor que el inicio en edades mayores a los 15 años.

El tener un ingreso mensual menor a 120 mil colones representa un 2.8 veces más probabilidad de tener sífilis materna en esta población.

La probabilidad de padecer de sífilis si no se tuvo control prenatal es de casi 5 veces mayor que en las mujeres que si tuvieron control.

La no planeación del embarazo representa 5 veces más probabilidad de padecer de sífilis que las mujeres que si planearon el embarazo.

La probabilidad de padecer de sífilis, si se usa drogas es de 12 veces mayor que las mujeres que no usaron drogas durante el embarazo.

El tener más de 3 compañeros sexuales presenta 3 veces más de probabilidad de padecer de sífilis que las que tuvieron menos de tres compañeros sexuales.

\section{DISCUSIÓN.}

Es importante el conocer los factores de riesgo, para poder evitar contagios materno y disminuir el índice de neonatos infectados en los centros de salud, ya que la transmisión de la sífilis de madre a hijo puede ocurrir en cualquier momento del embarazo. La tasa de transmisión vertical de la sífilis en mujeres no tratadas es de hasta un $80 \%$, dependiendo de la evolución de la infección materna. Este estudio permite apreciar que el $50 \%$ de las madres en fases primaria y secundaria $y$ el $30 \%$ en fases latente y terciaria dieron a luz a un niño prematuro. $\mathrm{La}$ sífilis durante el embarazo puede dar lugar a nacidos muertos, aborto espontáneo, retraso en el crecimiento intrauterino, o parto prematuro en un 50\% de los casos. La sífilis materna no tratada da lugar a muerte perinatal hasta en un $40 \%$ de los casos y, algunos autores sugieren que aún si la sífilis se adquirió durante los cuatro años previos al embarazo, es posible que pueda dar lugar a infección del feto en más del $70 \%$ de los casos.

Es importante recalcar que la investigación orienta a que aproximadamente el $52 \%$ de las muertes por sífilis congénita tienen lugar antes de las 30 semanas de la gestación. El tratamiento de la sífilis materna menos de 30 días antes del parto se considera inadecuado para prevenir la Sífilis Congénita en los niños y niñas. Por estas razones de morbimortalidad se hace necesario el conocimiento de Enfermería para aplicar los lineamientos de educación y prevención de la enfermedad entorno a esta problemática de salud.

El sujeto en este estudio, correspondió a las madres gestantes con sífilis materna y el objeto de conocimiento parte del estudio sobre los factores de riesgo de estas mujeres para padecer de sífilis e infectar a sus hijos, esta aproximación empírica se logra mediante tratamientos, 
teorías y dimensiones. Se intentó con este estudio que los conocimientos del objeto, trasciendan el sujeto, para que enfermería implemente planes de atención más personalizados y evitar futuros contagios de estas mujeres y sobre todo contribuir en la disminución de índices de morbimortalidad por sífilis congénita.

Como se evidenció anteriormente, se centró en una corriente epistemológica basada en el empirismo, ya que a partir del conocimiento que se adquiere en la formación académica profesional, sobre enfermedades de trasmisión sexual y de las actividades diarias en los servicios de neonatología y post parto de los centros de salud, se obtiene un conocimiento basado en la experiencia. Es decir se necesita una actividad cognitiva, como lo fue el análisis de estadísticas nacionales e internacionales, así como la valoración individual de las variables, para lograr una actividad práctica, donde se conozcan los objetos desde la experiencia y la visión.

Basado en un modelo de conocimiento empirista, donde solo el conocimiento sensible proporciona contacto con la realidad, y basado en enfermería como ciencia, se analizó la teorizante de Ramona $\mathrm{T}$ Mercer, quien plantea en su teoría, el proceso interactivo y de desarrollo que se produce a lo largo del tiempo en el cual la madre crea un vinculo con su hijo, aprende las tareas de cuidado del rol y expresa el placer y la gratificación con el rol. Dentro de sus conceptos, se encuentran la separación precoz materno filial, que es la separación de la madre después del momento del nacimiento a consecuencia de alguna enfermedad, esta separación provoca un estado de ansiedad en la madre, definido como: la característica de la persona propensa a percibir las situaciones estresantes como peligrosas o amenazantes y como un estado específico a la situación. También puede padecer de una serie de síntomas depresivos, y en particular, el componente afectivo del estado depresivo. Expone en su teoría que las enfermedades que pueden padecer la madre y el niño, interfieren en el proceso de unión, así mismo la familia como sistema dinámico, que incluye subsistemas (madre, padre, feto/niño) y grupos de pares (madre-padre, madrefeto/niño y padre-feto/niño), son importantes para un funcionamiento familiar de interrelación con las actividades de familia y subsistemas, en donde exista apoyo social, el cual incluye el apoyo emocional, informativo, físico y de valoración. (Ann Marriner, 1998).

Esta teoría es importante en este estudio, ya que el rol maternal se ve afectado e interrumpido durante el periodo de internamiento del niño y la niña, y es la familia quien debe dar apoyo a la madre durante esta etapa, en donde la madre experimenta una sensación de desconfianza y tristeza por su separación del bebé, hay una ruptura del binomio madre e hijo y padre hijo, que los afecta a todos durante el periodo de internamiento.

Con respecto a otro postulado de la autora, las percepciones de la madre y el padre acerca de su estado de salud anterior, actual y futura, la resistencia a la susceptibilidad de la enfermedad, la preocupación por la salud, la orientación de la enfermedad y el rechazo familiar del enfermo, hacen que los padres de los neonatos internado distorsionen su rol maternal, por sentirse muy culpables de no haber tenido una vida sexual sana, en donde no se contagiaran de sífilis, así mismo en muchos casos es hasta el momento del diagnóstico del neonato, 
cuando los padres se dan cuenta, por lo cual hay ruptura de relación marital o conyugal al dudar de su pareja, lo que afecta en gran medida el rol como padres que le puedan ofrecer al niño y niña enfermos.

Ramona $\mathrm{T}$ Mercer, plantea en su teoría conceptos importantes en torno al apoyo social, define el apoyo emocional como sentirse amado, cuidado, digno de confianza y comprendido, el apoyo informativo como la ayuda al individuo a solucionar problemas por si solo ofreciéndole útil información para cada problema o situación. Así mismo ven el apoyo físico como de tipo directo de ayuda, y el de valoración como la información que se está llevando a cabo en su rol, permite al individuo evaluarse a si mismo relación del rol de los demás.

Estos puntos con respecto al apoyo son esenciales para la presentación de estos resultados, ya que se aprecia que las madres necesitan de un apoyo continuo en todos estos aspectos, ya que muchas veces los factores socio demográficos, los hace carentes de conocimiento o existe presencia de violencia intrafamiliar agregada a la problemática de la enfermedad.

Se analizaron los ambientes en los cuales se desenvuelven las usuarias con este diagnóstico, mediante estadísticas y hojas de psicología, donde se evidenció que al igual que expone la Dra. Susan Vandale Toney y Dr. Felipe Uribe, del Centro de Investigaciones en Salud Pública, del Instituto Nacional de Salud Pública, de Cuernavaca, los factores prevalentes en la adquisición de sífilis materna radican en los contextos que vive la usuaria, ya que los estímulos negativos como tener un estatus económico bajo, una autoestima baja, el no tener pareja estable y un proveedor en algunos casos agresor, hace que las reacciones de adaptabilidad al entorno la exponga a vulnerabilidad de padecer a la enfermedad, contrario a lo presentado por el grupo control de mujeres quien en su mayoría presentaron estímulos positivos como pareja estable, desear el embarazo, no usar drogas, cuyas características hicieron adecuada la adaptabilidad al medio.

Al identificar los factores de riesgo más relevantes, se aprecia como la enfermedad de estas usuarias interviene en el proceso de salud y bienestar de sus hijos. (Gabbe, 2002), por lo que a pesar de ser antónimas, en este estudio están interrelacionadas de forma dinámica.

Se evidenció a través de revisión estadística que los índices de casos de sífilis congénita aumenta al pasar de los años, observándose que en el año del 2003 se presentaron 97 casos, en el 2004, 124 casos, en el 2005, 101 casos, en el año 2006, 108 casos y en el 2007, 133 casos, para un total de 656, de los cuales 415 fueron del sexo femenino y 241 masculinos, lo que evidencia que no es una enfermedad erradicada. (Control de Vigilancia y Epidemiología. Ministerio de Salud, 2006).

Esta enfermedad presentó una alta mortalidad, ya que solo en este Hospital en los meses de estudio, comprendidos de mayo a diciembre hubo 4 muertes por sífilis congénita, agregada, de un total de 27 muertes, según la unidad de estadísticas de este centro de salud, participante en la investigación.

Así mismo estudios revisados evidencian que si se ponen en práctica medidas que disminuyan los factores de riesgo maternos, y se mejora las condiciones de vida $\mathrm{y}$ salud de estas poblaciones, el 
número de casos de sífilis congénita disminuirá en gran medida, ya que no son hechos aislados, por que se necesita que la madre esté contagiada para que trasmita la enfermedad a su hijo, por ello solventando estas deficiencias, se mejoraría el régimen de salud de estos neonatos que son los que llevan la peor parte y las complicaciones más severas. (Cifuentes, 2004).

Se apreció que las condiciones principales de riego para padecer de sífilis materna, son las mismas en varios estudios analizados que las encontradas en esta investigación, por lo que se debe de dar seguimiento a estas causas ya probadas anteriormente. (Brunicardi y otros, 2006). Sin embargo confrontando el estudio realizado con opiniones de Profesionales en Enfermería, concuerdan con que, la principal causa para padecer sífilis por la madre es por medio de una vida promiscua, es decir tener muchos compañeros sexuales, o ser trabajadora de sexo comercial, así mismo concuerdan que el uso de drogas hace propensa a estas mujeres de padecer esta enfermedad. Y que según prioridades para padecer la enfermedad, se encuentran en el uso de drogas, el no tener control prenatal, $\mathrm{y}$ relaciones sexuales riesgosas, así mismo no consideran a la sífilis o sífilis congénita como una enfermedad mortal o peligrosa. Son pocos criterios los que proponen como factores de riesgo, el tener al compañero o pareja infectado y mucho menos los que creen que la falta de educación sexual puede hacer a una mujer contrayente de una enfermedad como la sífilis.

Según lo anterior se evidenció la necesidad de educación del personal de enfermería entorno a esta problemática, ya que según se analizó no es únicamente de mujeres promiscuas, sino actualmente afecta a amas de casa, por lo que se debe de quitar este estigma. Además la falta de educación sexual debería de ser el principal factor de riesgo, ya que una mujer bien informada es un ente de cambio y prevención, y dentro de esta educación se incluyen algunas respuestas dadas como el uso de métodos preventivos, y conocer a la pareja sexual. A pesar de que es el profesional de enfermería quien mejor informado entorno a esta problemática debe de estar, posee dudas sobre la raíz primordial del asunto, por lo cual necesita capacitarse para poder ofrecer una atención holística. Esta situación confrontada con el estudio realizado por médicos colombianos difieren de nuestra población de estudio, ya que nuestra población femenina infectada, son mujeres amas de casa, en donde la infección radica por medio de contagio con su pareja estable, no por promiscuidad de ellas, las cuales la mayoría de las veces se da cuenta de su enfermedad hasta el momento del embarazo o parto, cargando con un prejuicio social, y de culpabilidad por contagiar a su hijo.

El Dr. Solón Chavarría Aguilar, de la Unidad de Control y Prevención del Sida de la Caja Costarricense de Seguro Social, considera:

"A la luz de la cantidad de niños
enfermos, existe un número
importante de mujeres y hombres con
sífilis, que no se detectan y tampoco se
reportan a la base del Ministerio de
Salud como lo dicta la Ley."

Esto hace que se tengan sub registros de la enfermedad, y no se cuenten con estadísticas totales de este fenómeno epidemiológico, así mismo se aprecia que son nuestros y nuestras adolescentes quienes mayormente se encuentran 
contagiados, por su inexperiencia en asuntos de sexualidad, lo que aunado a una deplorable educación sexual, contribuyen a aumentar el índice de contagios. Por ello se debe entender que la sífilis congénita es la forma más seria de la enfermedad. Muchos casos terminan en un aborto espontáneo o un nacido muerto y los que sobreviven pueden tener daño en el cerebro, hígado, tejido óseo y corazón. El bebé puede sanar de la sífilis, pero la secuela lo puede incapacitar de por vida.

\section{CONCLUSIONES.}

Las condiciones sociodemográficas estudiadas influencian notablemente la presencia de sífilis materna y por consiguiente a la sífilis congénita por lo cual: dentro de los factores de riesgo generales se encuentran más vulnerables las mujeres que viven en Desamparados, específicamente en Patarra, así como aquellas que son menores de edad y además primigestas.

Dentro de los factores sociales, las inequidades de género se reflejan en el proceso de transmisión de la infección, ya que la mayor vulnerabilidad está determinada por una cultura que limita el acceso a bienes y servicios a la población femenina y les impide tomar decisiones sexuales y reproductivas autónomas e informadas. El hecho de que es común que en muchos casos sean sus compañeros o esposos quienes deciden cuando, como y con qué frecuencia tener relaciones sexuales limita las posibilidades de que las mujeres pongan en práctica medidas de prevención o protección para no infectarse. Son estas asimetrías de poder las que se determinan la mayor vulnerabilidad social de las mujeres.
Dentro de los factores de riesgo culturales y personales, la falta de educación de estas madres en todo aspecto desde conocimientos básicos, hasta la falta de educación sexuales, hacen que esta mujeres vivan en un mundo de desconocimiento, que repercute en la no elección de métodos anticonceptivos, en el inicio temprano de sexualidad, en no tener control prenatal, en el uso de drogas, esto a su vez predispone a la pobreza, ya que no existe el suficiente ingreso para subsidiar sus gastos y los de sus hijos. (Olds, 1995).

\section{BIBLIOGRAFIA.}

Alburg. S.A. (2008). La Guía Completa de la Salud. [Documento en línea] Disponible: http://www.latinsalud.com. [Consulta: 2008, Marzo 10].

Barrantes, Echeverría (1999). Investigación un camino al conocimiento. San José: EUNED.

Calvo Neyssa. (2005). Sífilis toca a los bebes desde el vientre. San José. 03-set-2005: Periódico al día.

Brunicardi F, Schwartz y otros. (2006). Principios de Cirugía. México: D.F. : Ed. El Ateneo.

Cifuentes R. (2004). Texto de Obstetricia y Ginecología. Sociedad Colombiana de Obstetricia. Bogotá: Ed. Médica.

Control de Epidemiología y Vigilancia del Ministerio de Salud. (2006). Registros estadísticos. San José: Ed. Ministerio de Salud de Costa Rica.

Dever, A. (1991). Epidemiología y administración de servicios de salud: Organización Panamericana de la Salud. Santiago: Ed. Aspen Publisher.

Gabbe, S, Niebyl, J., Simpson, J. (2002). Obstetrics Normal and Problem Pregnancies. Michigan: Ed. Harcourt.

Hernández Sampierie, Fernández C, Baptista L, (1998). Metodología de la investigación. Mèxico.D. F.: Ed. Mac Graw Hill. 
Julia Valderama (2003). Eliminación de la Sífilis Congénita en América Latina y el Caribe. Organización Panamericana de la Salud: Bogotá: Ed. Aspen Publisher.

Ann Marrier, Martha Raile (1998). Modelos y teorías en Enfermería. Madrid: Ed. Harcourt.

Kumar, Cotran, Robbins. (2003). Patología Humana. Florida: Ed. McGraw Hill.

Lowdermilk, D; Perry, S y Bobak, I (2003) Enfermeria Materno Infantil. Madrid. Ed. Harcourt/ Océano.

Lukehart SA, Holmes KK. (2005). Principios de Medicina Interna. Kansas: Ed. Harrison.

Olds, S; London, M y Ladewig, P (1995) Enfermería Materno Infantil. México D. F: Ed. McGraw-Hill.

Organización Panamericana de la Salud. (2004) Promoción de la salud/OPS Indicadores de Salud y Bienestar en Municipios. Washington: D.C. Ed.OPS.

Orozco, B. (2003). La sífilis y Enfermedades de transmisión sexual. Bogotá: Ed. Díaz F, Ospina S.

Schwarcz, R., (2005). Obstetricia. Buenos Aires: Ed. El Ateneo.

Susan Vandale. (2003). Factores para adquisición de sífilis materna. Cuernavaca: Ed. Centro de Investigaciones en Salud Pública, del Instituto Nacional de Salud Pública.

Varney H., (2006). Partería Profesional de Varney. México: DF. Ed. Organización Panamericana de la Salud. 